\title{
Kinetics of Diffusion-Controlled Annihilation with Sparse Initial Conditions
}

\author{
E. Ben-Naim ${ }^{1}$ and P. L. Krapivsky ${ }^{2}$ \\ ${ }^{1}$ Theoretical Division and Center for Nonlinear Studies, \\ Los Alamos National Laboratory, Los Alamos, New Mexico 87545, USA \\ ${ }^{2}$ Department of Physics, Boston University, Boston, Massachusetts 02215, USA
}

\begin{abstract}
We study diffusion-controlled single-species annihilation with sparse initial conditions. In this random process, particles undergo Brownian motion, and when two particles meet, both disappear. We focus on sparse initial conditions where particles occupy a subspace of dimension $\delta$ that is embedded in a larger space of dimension $d$. We find that the co-dimension $\Delta=d-\delta$ governs the behavior. All particles disappear when the co-dimension is sufficiently small, $\Delta \leq 2$; otherwise, a finite fraction of particles indefinitely survive. We establish the asymptotic behavior of the probability $S(t)$ that a test particle survives until time $t$. When the subspace is a line, $\delta=1$, we find inverse logarithmic decay, $S \sim(\ln t)^{-1}$, in three dimensions, and a modified power-law decay, $S \sim(\ln t) t^{-1 / 2}$, in two dimensions. In general, the survival probability decays algebraically when $\Delta<2$, and there is an inverse logarithmic decay at the critical co-dimension $\Delta=2$.
\end{abstract}

Reaction-diffusion processes are ubiquitous in a wide range of natural and physical phenomena [1-3]. Minimal models of diffusion-limited aggregation, coalescence, and annihilation play a central role in the theory of fractals 4, pattern formation [5, 6, and non-equilibrium statistical physics [7]. In the latter context, the central finding is that in low dimensions, spatial correlations dominate and lead to slow reaction kinetics. This behavior has been observed experimentally [8 10, and it well understood theoretically [11 22 .

The single-species annihilation process has played a key role in illuminating the statistical mechanics of reaction kinetics. It is represented by the reaction scheme

$$
A+A \rightarrow \emptyset .
$$

In this random process, particles undergo ordinary diffusion and whenever two particles meet, they both disappear. Starting with a uniform density, the survival probability of a test particle, $S(t)$, exhibits the long-time asymptotic behaviors [12, 15, 16.

$$
S(t) \sim \begin{cases}t^{-d / 2} & d<2 \\ (\ln t) t^{-1} & d=2 \\ t^{-1} & d>2 .\end{cases}
$$

The decay is slow below the critical dimension, while in the complementary case, there is a quick decay, and the exponent does not depend on the dimension. Strong spatial correlations in the positions of the surviving particles, which develop below the critical dimension $d_{c}=2$, are responsible for this behavior [12, 23, 24.

Previous studies of single-species diffusion-controlled annihilation have mostly dealt with spatiallyhomogeneous situations where the initial density is uniform. Among a few exceptions are the analyses of single-species reaction processes in one dimension where particles initially occupy the half-line [20, 21, 25, 26. There are also a few studies of single-species annihilation with a finite (but large) number of particles [27, 28.

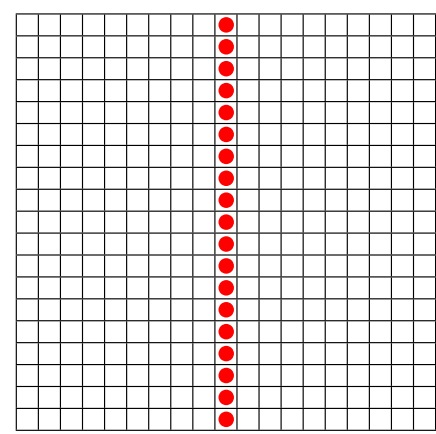

FIG. 1: An initial conditions where particles, denoted by red dots, occupy a line in two dimensions $(\delta=1, d=2)$.

A recent study shows that the survival probability is altered dramatically when the initial number of particles is finite, as a finite number of the particles may survive indefinitely 29 .

Motivated by these results, we consider reaction kinetics of single-species annihilation with another sparse initial condition. Specifically, we consider beginning configurations where particles occupy a subspace with dimension $\delta$ that is embedded in a space with larger dimension $d$. We consider the simplest possible case where the initial distribution of particles is uniform inside the occupied sub-space (figure 1 illustrates a line in two dimensions).

Our main result is that the co-dimension $\Delta=d-\delta$ controls the asymptotic behavior. For dimensions $d>2$, we obtain

$$
S(t) \sim \begin{cases}t^{-(2-\Delta) / 2} & \Delta<2 \\ (\ln t)^{-1} & \Delta=2, \\ S_{\infty}+\text { const. } \times t^{-(\Delta-2) / 2} & \Delta>2 .\end{cases}
$$

All particles disappear when the co-dimension is sufficiently small, $\Delta<2$, and the survival probability decays as a power law in this case. A finite fraction 
$S_{\infty} \equiv S(\infty)$ of the particles survives indefinitely when the co-dimension is large enough, $\Delta>2$. Finally, all particles disappear in the borderline case $\Delta=2$, although the survival probability decays extremely slowly, as inverse the logarithm of time.

The single-species annihilation process is tractable analytically only in one dimension (see e.g. $16,22,24,26$ ). Therefore our arguments in favor of (3) rely on heuristic reasoning. We start with three dimensions, $d=3$, and derive (3) for initial conditions where particles initially occupy a line, $\delta=1$, or a plane, $\delta=2$. We then analyze a line, $\delta=1$, embedded in a two-dimensional space, $d=2$. We use scaling methods to obtain $(3)$ from the reaction-diffusion equation, and thereby adopt a similar approach to that used to analyze initial conditions with a finite number of particles 29.

In single-species annihilation, identical particles undergo diffusion and whenever two particles touch each other, both particles disappear. This process can be realized in discrete or in continuum space. In the discrete version, particles move in an unbounded hyper-cubic lattice of dimension $d$, and each lattice site can be occupied by at most one particle. Particles move asynchronously and independently. In each hoping event, a particle moves to one of its $2 d$ neighboring sites, chosen at random. If the particle lands on an occupied site, both particles disappear. Initially, the particles occupy a subspace of dimension $\delta$. We implemented this discrete version in our numerical simulations.

We begin with the three-dimensional case, $d=3$, and our starting point is the standard reaction-diffusion equation for the density $c \equiv c(x, y, z, t)$

$$
\frac{\partial c}{\partial t}=D \nabla^{2} c-K c^{2}
$$

Here $D$ is the diffusion coefficient, $\nabla^{2}$ the Laplace operator, and $K$ the reaction coefficient. The reaction term is quadratic in $c$ reflecting that the reaction process (1) involves two particles [30]. For a spatially uniform system, the diffusion term vanishes, so (4) reduces to $d c / d t=-K c^{2}$, and the density decay $c \sim t^{-1}$ in (2) follows. Of course, for homogeneous systems, the survival probability of a test particle is proportional to the density, $S \propto c$. Here, and in the rest of this study, we set the diffusion coefficient and the reaction coefficient to unity $D=K=1$, as we are primarily interested in asymptotic behaviors.

We now treat the case where the particles occupy a line, $\delta=1$. Without loss of generality we set this line as the $z$-axis in a standard Cartesian coordinate system. With this setup, we expect that $\partial_{z} c=0$. Further, the Laplace operator in (4) becomes two-dimensional, $\nabla^{2} \equiv \partial_{x}^{2}+\partial_{y}^{2}$. The density $c \equiv c(x, y, t)$ depends only on two of the three coordinates, and the survival proba-

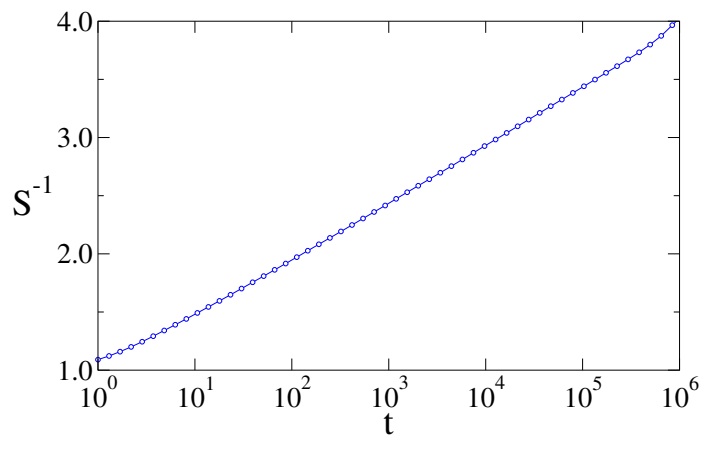

FIG. 2: The inverse survival probability $S^{-1}$ versus time $t$ for single-species annihilation in three dimension, starting with a line of particles $(\delta=1)$. The simulations were performed on a cubic lattice with $2500^{3}$ sites and periodic boundary conditions. Initially, there are 2500 particles.

bility of a particle is the integrated density

$$
S(t)=\iint d x d y c(x, y, t)
$$

We stress that the survival probability equals the total number of particles per unit length along the $z$-axis. By substituting (5) into the reaction-diffusion equation (4), we find that the survival probability obeys

$$
\frac{d S}{d t}=-\iint d x d y c^{2} .
$$

The right-hand side of this equation is simply the total reaction rate per unit length.

We now use heuristic methods to estimate the total reaction rate in (6). The initial arrangement of the particles is radially-symmetric along the $z$-axis, and this symmetry is maintained throughout the reaction-diffusion process. Therefore, the density depends only on the radial coordinate, $c \equiv c(r, t)$ with $r=\sqrt{x^{2}+y^{2}}$. Since particles move diffusively, we expect that particles are essentially confined to within a cylinder of growing radius $r \sim \sqrt{t}$ whose axis coincides with the $z$-axis. Hence, we approximate the density by

$$
c(r, t) \sim \frac{S(t)}{t} \times \begin{cases}1 & r<\sqrt{t} \\ 0 & r>\sqrt{t} .\end{cases}
$$

In this approximation, the density is uniform inside the growing cylinder and vanishes outside of it. By substituting the approximate density (7) into (6), we obtain a rate equation for the survival probability,

$$
\frac{d S}{d t} \sim-\frac{S^{2}}{t} .
$$

In the long-time limit, the survival probability is inversely proportional to the logarithm of time,

$$
S \sim(\ln t)^{-1} .
$$




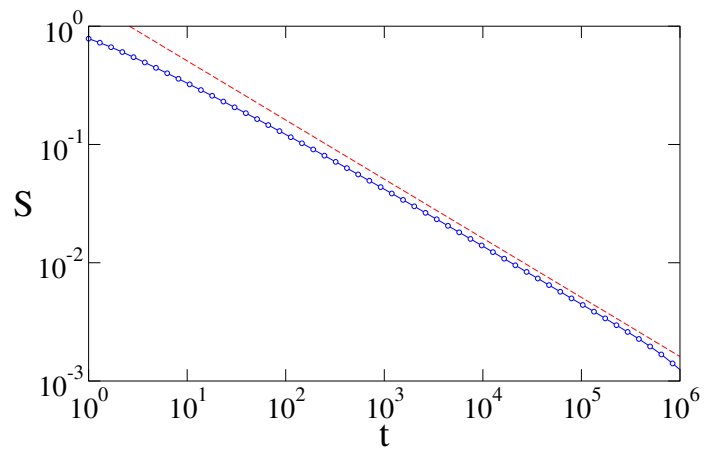

FIG. 3: The survival probability $S(t)$ versus time $t$ for $d=3$ and $\delta=2$. The simulations were performed using a cubic lattice with $2500^{3}$ sites and periodic boundary conditions. Initially, $2500^{2}$ particles occupy a plane.

This extremely slow decay demonstrates how sparse initial condition can result in a slow reaction process. The logarithmic time dependence 9 is difficult to confirm numerically, but results of our large-scale simulations using a cubic lattice with $2500^{3}=1.5625 \cdot 10^{10}$ sites support the theoretical prediction (Fig. 22).

We now consider a plane, $\delta=2$, embedded in threedimensional space. The co-dimension now equals unity, $\Delta=d-\delta=1$, and the Laplace operator is effectively one-dimensional, $\nabla^{2} \equiv \partial_{x}^{2}$. By repeating the steps leading to (8), we obtain

$$
\frac{d S}{d t} \sim-\frac{S^{2}}{\sqrt{t}}
$$

Therefore, the survival probability has the power-law decay, $S \sim t^{-1 / 2}$ as stated in (3). Results of our numerical simulations are in good agreement with this theoretical prediction (Fig. 3).

Equations (6) and (10) can be easily generalized to arbitrary co-dimension $\Delta$. The general rate equation reads $d S / d t \sim-S^{2} / t^{\Delta / 2}$ and equation (3) follows. When the co-dimension is large enough, $\Delta>2$, the survival probability saturates at a constant value and consequently, a test particle has a finite chance of avoiding the annihilation process. In the complementary case, $\Delta<2$, the density decays as a power law indefinitely.

Next, we discuss the behavior at the critical dimension, $d=2$. When $d>2$, the reaction rate in (4) is a constant, while for $d<2$ the reaction coefficient scales as a power of the density, $K \sim c^{(2-d) / d}$ 24]. At the critical dimension, the effective reaction coefficient decays as a logarithmic function of the density, $K \sim 1 / \ln (1 / c)$. Therefore

$$
\frac{\partial c}{\partial t}=\nabla^{2} c-\frac{c^{2}}{\ln (1 / c)} .
$$

For uniform systems, the diffusion term vanishes, and equation (11) simplifies to $d c / d t \sim-c^{2} / \ln (1 / c)$. The reduced reaction rate leads to a logarithmic enhancement in the survival probability $\sim(\ln t) t^{-1}$ stated in (2).

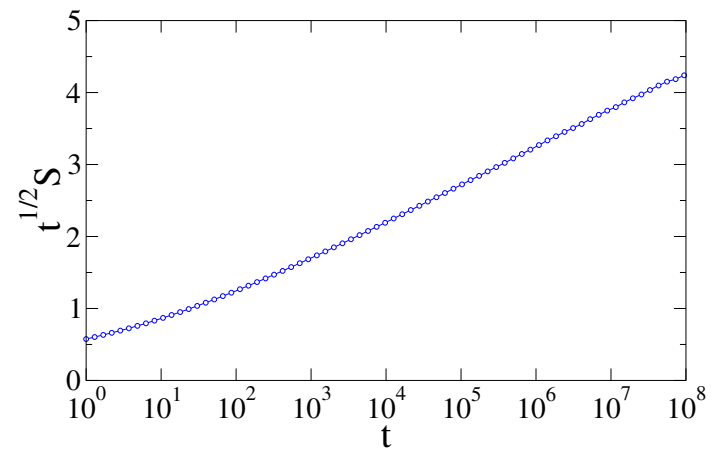

FIG. 4: Plot of $\sqrt{t} S(t)$ versus $t$ for the two-dimensional singlespecies annihilation process with initially occupied line. The simulations were performed on a square lattice with $125000^{2}$ sites and periodic boundary conditions, that is initially occupied by 125000 particles.

We now turn to a line in two dimensions as illustrated in Fig. 1. In a Cartesian coordinate system, we envision the particles to occupy the $y$-axis. Now, the density is uniform along the $y$-direction and the Laplace operator in (11) is effectively one-dimensional, $\nabla^{2} \equiv \partial_{x}^{2}$. By integrating Eq. (11), we see that the survival probability, $S(t)=\int d x c(x, t)$, decays according to

$$
\frac{d S}{d t}=-\int d x \frac{c^{2}}{\ln (1 / c)}
$$

Once again, the total reaction rate represents a rate per unit length along a line parallel to the $y$-axis. We anticipate that the reaction process is limited to the diffusive region $|x|<t^{1 / 2}$, and using the same reasoning behind (6), we obtain a rate equation for the survival probability,

$$
\frac{d S}{d t} \sim-\frac{S^{2}}{t^{1 / 2} \ln \left(t^{1 / 2} / S\right)} .
$$

Therefore, the survival probability decays according to

$$
S \sim(\ln t) t^{-1 / 2} .
$$

Results of our numerical simulations are in good agreement with this prediction (Fig. 4).

We now discuss the complementary case where particles occupy a sub-space with co-dimension $\Delta$ at or below the critical dimension, $d \leq 2$. In two dimensions, the effective Laplace operator has dimension $\Delta$, and the quantity $t^{1 / 2}$ in the denominator of equation (13) should be replaced with $t^{\Delta / 2}$. Below the critical dimension, $d<2$, we substitute the effective reaction coefficient $K \sim c^{(2-d) / d}$ into (4). The survival probability exhibits the asymptotic decay

$$
S \sim \begin{cases}(\ln t) t^{-\delta / 2} & d=2 \\ t^{-\delta / 2} & d<2\end{cases}
$$


In our numerical studies, we used a standard algorithm for simulating the annihilation process [24]. Particles reside in a $d$-dimensional hyper-cubic lattice with linear dimension $L$ and periodic boundary conditions. Initially, $L^{\delta}$ particles occupy a hyper-cubic lattice with dimension $\delta$, that is embedded in the larger space, while the rest of the sites are empty. In each elementary simulation step one particle, chosen at random, moves to one of its neighboring sites. If that site is occupied, both particles are removed from the system. Time is augmented by the inverse of the number of remaining particles. The system memory required by this algorithm is $\mathcal{O}\left(L^{d}\right)$, and the processing cost per unit time is proportional to the number of surviving particles, $S(t) \times L^{\delta}$.

In what follows, we show how several of the above results can be derived using alternative probabilistic arguments. First, we obtain the asymptotic decay (14) using such methods. To derive the $S \sim(\ln t) t^{-1}$ decay in $(2)$, one begins with the number of distinct lattice sites visited by a random walker 31] in two dimensions,

$$
\mathcal{N} \sim \frac{t}{\ln t}
$$

Then one argues that if all lattice sites are initially occupied, all particles in a visited region coalesce into one particle, and the density is reciprocal to this number, $S \sim \mathcal{N}^{-1}$, in agreement with 2 . When the particles initially occupy a line, we can focus on a square domain with area $\sqrt{t} \times \sqrt{t}$. We now replace the inhomogeneous spatial distribution with a homogeneous distribution with density $t^{-1 / 2}$, such that the total initial number of particles equals the linear dimension of the domain, $\sqrt{t}$. The total number of particles in the visited region scales as $t^{-1 / 2} \mathcal{N}$, and its reciprocal gives (14).

Another key result is that when the co-dimension $\Delta>2$, each particle has a finite probability $S_{\infty}>0$ of surviving the annihilation process. This result can also be derived using probabilistic arguments [29, as we now demonstrate for a line, $\delta=1$, embedded in a $d$ dimensional space. We consider the annihilation to take place in an unbounded hypercubic lattice where the line $(0, \ldots, 0, n)$ for all $-\infty<n<\infty$ is initially fully occupied while the rest of the lattice is empty. We also denote by $P_{n}$ the probability that two random walkers, separated by distance $n$ in a $d$-dimensional space, never meet. The probability $S_{\infty}$ that the particle, initially located at the origin, survives forever has the lower bound,

$$
S_{\infty}>\prod_{n \geq 1} P_{n}^{2}
$$

Here, we used $P_{n}=P_{-n}$. If this product is finite, then the survival probability is necessarily finite. We now invoke the well-known asymptotic behavior [32],

$$
1-P_{n} \sim n^{-(d-2)},
$$

when $n \gg 1$, to show that the infinite product in 17 converges to a finite value when $d>3$. The above analysis can also be repeated for a plane, $\delta>2$, and in this case the lower bound is finite when $d>4$. Hence, the survival probability is finite when the co-dimension is larger than the critical value, $\Delta>2$, in agreement with (3).

In summary, we have studied kinetics of a singlespecies reaction process, starting with sparse initial conditions. In our setup, particles initially occupy a subspace with dimension $d-\Delta$ embedded in a $d$-dimensional space. The effective dimension of the diffusion operator equals the co-dimension $\Delta$, and this parameter controls the asymptotic behavior. The survival probability of a particle is finite above the critical co-dimension, $\Delta>2$, but it vanishes otherwise. We obtained the timedependent behavior of the fraction of surviving particles using scaling methods. While our scaling analysis and numerical simulations were performed for integer dimensions and co-dimensions, we expect that the results extend to non-integer dimensions or co-dimensions. Our investigation complements studies of catalytic reactions [33, and reactions with mobile and immobile particles 34 with a similar geometry.

The survival probability (3) generalizes a classic result in probability theory. To see this, let us consider two random walkers in $d$ dimensions, which formally corresponds to the case $\delta=0$. The separation between these two particles performs a random walk as well, and the survival probability equals the probability that an ordinary random walk has yet to visit the origin. Indeed, by substituting $\Delta=d$ into (3), we recover the Pólya theorem [35]. Hence, our main result (3) generalizes the Pólya theorem from a single random walk to a system of infinitely many random walkers that interact through the annihilation process.

There are a number of natural extensions of this work. An immediate generalization is to aggregation processes. The aggregate mass should no longer be uniform in space, and it is natural to investigate the spatial dependence of the mass distribution. It would also be interesting to study the two-species annihilation process $A+B \rightarrow \emptyset$ under sparse initial conditions. For this reaction scheme, starting from homogeneous initial conditions, the critical dimension is higher, $d=4$ [11]. Our study finds that the critical co-dimension is $\Delta=2$, and hence, it is plausible that there are multiple regimes of behavior for reactions involving multiple species.

We acknowledge support from the US-DOE grant DEAC52-06NA25396 (EB) and the BSF Grant No. 2012145 (PLK).

[1] P. Grindrod, Patterns and Waves: The Theory and Applications of Reaction-Diffusion Equations (Clarendon 
Press, 1991).

[2] S. K. Frielander, Smoke, Dust and Haze: Fundamentals of Aerosol Behavior (Wiley, New York, 1977).

[3] A. A. Ovchinnikov, S. F. Timashev and A. A. Belyi, Kinetics of Diffusion Controlled Chemical Processes (Nova Science Pub. Inc., 1989).

[4] T. A. Witten and L. M. Sander, Phys. Rev. Lett. 47, 1400 (1981).

[5] M. Cross and H. Greenside, Pattern Formation and Dynamics in Nonequilibrium Systems (Cambridge University Press, Cambridge, 2009).

[6] W. van Saarloos, Phys. Rep. 386, 29 (2003).

[7] U. C. Tauber, M. Howard, and B. P. Vollmayr-Lee, J. Phys. A 38, R79 (2005).

[8] R. Kroose, H. Fleurent, and R. Sprink, Phys. Rev. E 47, 2462 (1993).

[9] R. M. Russo, E. J. Mele, C. L. Kane, I. V. Rubtsov, M. J. Therien, and D. E. Luzzi, Phys. Rev. B 74, 041405 (2006).

[10] J. Allam, M. T. Sajjad, R. Sutton, K. Litvinenko, Z. Wang, S. Siqqique, Q. H. Yang, W. H. Loh, and T. Brown, Phys. Rev. Lett. 111, 197401 (2013).

[11] Ya. B. Zeldovich and A. A. Ovchinnikov, Chem. Phys. 28, 215 (1978).

[12] M. Bramson and D. Griffeath, Z. Wahrsch. Verw. Geb. 53, 183 (1980).

[13] D. Toussaint and F. Wilczek, J. Chem. Phys. 78, 2642 (1983).

[14] P. G. de Gennes, J. Chem. Phys. 76, 3316 (1982).

[15] K. Kang and S. Redner, Phys. Rev. A 32, 435 (1985).

[16] D. C. Torney and H. M. McConnell, J. Chem. Phys. 87, 1941 (1983).

[17] J. L. Spouge, Phys. Rev. Lett. 60, 871 (1988).

[18] B. R. Thomson, J. Phys. A 22, 879 (1989).
[19] S. F. Burlatsky, A. A. Ovchinnikov, and G. S. Oshanin, Sov. Phys. JETP 68, 1153 (1989)

[20] D. ben-Avraham, M. A. Burschka, and C. R. Doering, J. Stat. Phys. 60, 695 (1990).

[21] D. ben-Avraham, Phys. Rev. Lett. 81, 4756 (1998).

[22] T. O. Masser and D. ben-Avraham, Phys. Rev. E 64, 062101 (2001).

[23] D. ben-Avraham and S. Havlin, Diffusion and Reactions in Fractals and Disordered Systems (Cambridge University Press, Cambridge, 2000).

[24] P. L. Krapivsky, S. Redner and E. Ben-Naim, A Kinetic View of Statistical Physics (Cambridge University Press, Cambridge, 2010).

[25] L. Frachebourg, P. L. Krapivsky, and S. Redner, J. Phys. A 31, 2791 (1998).

[26] P. L. Krapivsky and E. Ben-Naim, J. Stat. Mech. P05003 (2015).

[27] K. Krebs, M. P. Pfannmuller, B. Wehefritz, and H. Hinrichsen, J. Stat. Phys. 78, 1429 (1995).

[28] G. Foltin, K. A. Dahmen, and N. M. Shnerb, arXiv:condmat/0005015

[29] E. Ben-Naim and P. L. Krapivsky, arXiv:1605.07238.

[30] M. V. Smoluchowski, Z. Phys. Chem. 92, 129 (1917); ibid 92, 155 (1917).

[31] P. Mörters and Y. Peres, Brownian Motion (Cambridge: Cambridge University Press, 2010).

[32] S. Redner, A Guide to First-Passage Processes (Cambridge University Press, Cambridge, 2001).

[33] G. Oshanin and A. Blumen, J. Chem. Phys. 108, 1140 (1998).

[34] E. Ben-Naim, Phys. Rev. E 53, 1566 (1996).

[35] G. Pólya, Math. Ann. 84, 149 (1921). 